ISSN 2590-9770

The Art of Discrete and Applied Mathematics 2 (2019) \#P2.03

https://doi.org/10.26493/2590-9770.1342.109

(Also available at http://adam-journal.eu)

\title{
Groupoids on a skew lattice of objects
}

\author{
Desmond G. FitzGerald * \\ School of Mathematics and Physics, University of Tasmania, \\ Private Bag 37, Hobart 7001, Australia \\ To Jonathan Leech-Na zdravje!
}

Received 16 October 2018, accepted 11 December 2019, published online 27 December 2019

\begin{abstract}
Motivated by some alternatives to the classical logical model of boolean algebra, this paper deals with algebraic structures which extend skew lattices by locally invertible elements. Following the meme of the Ehresmann-Schein-Nambooripad theorem, we consider a groupoid (small category of isomorphisms) in which the set of objects carries the structure of a skew lattice. The objects act on the morphisms by left and right restriction and extension mappings of the morphisms, imitating those of an inductive groupoid. Conditions are placed on the actions, from which pseudoproducts may be defined. This gives an algebra of signature $(2,2,1)$, in which each binary operation has the structure of an orthodox semigroup. In the reverse direction, a groupoid of the kind described may be reconstructed from the algebra.
\end{abstract}

Keywords: Inductive groupoids, skew lattices, orthodox semigroups.

Math. Subj. Class.: 20L05, 20M19, 06B75

\section{Non-commutative and non-idempotent lattice analogues}

As non-classical logics have been developed for various knowledge domains, so various algebras have been proposed as extensions or alternatives to the classical model of boolean algebra. A significant one for this paper is the theory of skew lattices; for a contemporary account, see Leech's surveys [10,11]. We provide the details of our notation in Section 3. Another proposal is that of $\mathrm{MV}$-algebras, and their coördinatisation via inverse semigroups as described by Lawson and Scott [9]. Thus one theme is to allow sequential operations and hence non-commutative logical connectives, and another introduces non-idempotent

\footnotetext{
* The author thanks Vicky Gould, Michael Kinyon and Anja Kudryavtseva for their interest and input in discussions, both in person and by email, which helped convert a half-baked workshop idea into an interesting project.

E-mail address: d.fitzgerald@utas.edu.au (Desmond G. FitzGerald)
} 
connectives. This note will consider a combination of these themes, by seeking reasonable structures which extend skew lattices by locally invertible elements.

The principal tool in this construction is based on the ideas behind the EhresmannSchein-Nambooripad (ESN) theorem, of which a full account is given in Chapter 4 of Lawson's book [8]: we consider a small category of isomorphisms in which the set of objects carries the structure of a skew lattice. We postulate that the objects act (partially) on the morphisms by left and right restriction and extension mappings of the morphisms, imitating those of an inductive groupoid. Certain reasonable conditions are postulated, and from these a suitable pseudoproduct is defined, much as in the inverse semigroup case, for each skew lattice operation (a non-commutative "meet" and "join"). This results in a total algebra involving two orthodox semigroups with a common set of idempotents isomorphic to the given skew lattice.

Because of the complexity involved in having two operations, we begin by considering a groupoid over a set of objects with a single band operation. A much more general situation has been studied, under the name of weakly B-orthodox semigroups, by Gould and Wang in [2], but because the present author has been unable to find this special case treated in the literature, a detailed account will be given here. Later sections deal with the pair of linked band operations, construct the total algebra described above, and show how the original groupoid may be recovered from the algebra. Aspects of the constructions which need further elaboration are noted in the final section.

\section{Groupoids on a band of objects}

Let us recall from [8] that an inverse semigroup is equivalent to an inductive groupoid, i.e.,

- a (small) category of isomorphisms with

- a meet operation on objects and

- a notion of restriction of a morphism to any of its subdomains.

We attempt something similar here, but changing the conditions on the set of objects. Let $\mathscr{G}$ be a groupoid with composition $\circ$ and $B$ its set of objects, endowed with an associative and idempotent operation $\wedge$. Then $(B, \wedge)$ is known as a lower band, and possesses a pair of natural preorders: we write

- $a \leq_{L} b$ if and only if $a=a \wedge b$, and $a \leq_{R} b$ if and only if $a=b \wedge a$.

As usual, we may identify each object $b$ with its identity $\mathbf{i}_{b}$, and write $\mathbf{d} g$ and $\mathbf{r} g$ for the domain and range maps in $\mathscr{G}$, thus: $\mathbf{d} g=g \circ g^{-1}, \mathbf{r} g=g^{-1} \circ g$. Suppose too that for each $a \in B$ there are left and right restriction (partial) operations ${ }_{a}|,|_{a}: \mathscr{G} \rightarrow \mathscr{G}$ such that:

- ${ }_{a} \mid g$ is defined whenever $a \leq_{L} \mathbf{d} g$, with ${ }_{a} \mid g: a \rightarrow \mathbf{r}\left({ }_{a} \mid g\right) \leq_{L} \mathbf{r} g$;

and (lateral-) dually,

- $\left.g\right|_{a}$ is defined whenever $a \leq_{R} \mathbf{r} g$, with $\left.g\right|_{a}: \mathbf{d}\left(\left.g\right|_{a}\right) \rightarrow a, \mathbf{d}\left(\left.g\right|_{a}\right) \leq_{L} \mathbf{d} g$.

Figure 1 shows the left and right restrictions. (There are analogous (in fact, vertically dual) requirements for extension operators, which will be dealt with more explicitly in Section 3.) Certain sensible axioms must be satisfied: 

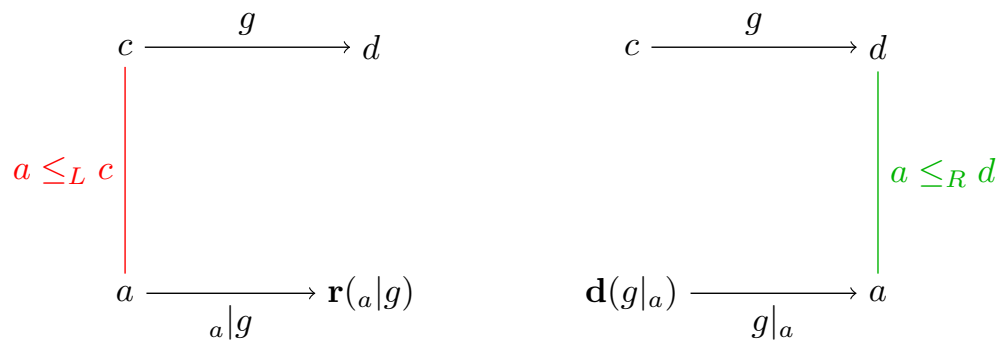

Figure 1: Left and right restriction operators.

(i) (identities) $\quad \mathbf{d}(g) \mid g=g$;

(ii) (preorders) if $a \leq_{L} b$, then ${ }_{a} \mid \mathbf{i}_{b}=\mathbf{i}_{a}$;

(iii) (transitivity) if $a \leq_{L} b \leq_{L} \mathbf{d} g$, then ${ }_{a}\left|g={ }_{a \wedge b}\right| g={ }_{a} \mid\left({ }_{b} \mid g\right)$;

(iv) (composition) if $f \circ g$ is defined (so that $\mathbf{r} f=\mathbf{d} g$ ), then

$$
\left.{ }_{a}\left|(f \circ g)=\left({ }_{a} \mid f\right) \circ\left(\mathbf{r}_{(a} \mid f\right)\right| g\right),
$$

the right-hand composite being defined because $\mathbf{r}\left({ }_{a} \mid f\right) \leq_{L} \mathbf{r} f=\mathbf{d} g$; see Figure 2 .

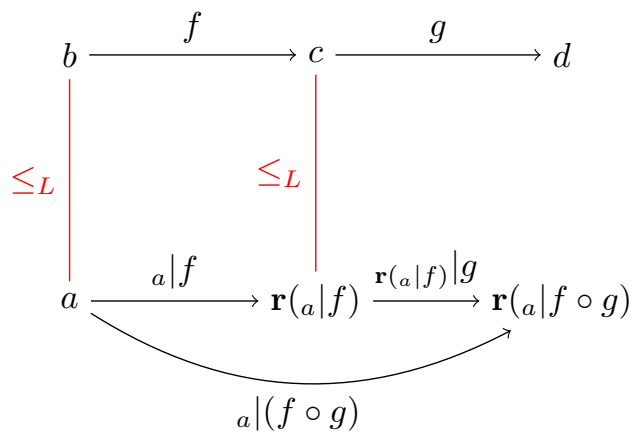

Figure 2: Restriction of a composite morphism.

\subsection{Actions and conjugacy}

Let us write, without prejudice, $a^{g}$ as an alternative for $\mathbf{r}\left({ }_{a} \mid g\right)$, and ${ }^{g} a$ for $\mathbf{d}\left(\left.g\right|_{a}\right)$. This lightens the notation, and emphasises the similarity to actions and conjugates. Caution: However, it should not be taken to mean that anything like $a^{f}=\left(\left.f^{-1}\right|_{a}\right) \circ(a \mid f)$, or $a^{f}=f^{-1} a$, or $a|f=f|_{a^{f}}$ necessarily hold: in general, $\left.f\right|_{a^{f}}=\left.f\right|_{\mathbf{r} f \wedge a^{f}}$.

What we do have, following from ${ }_{a} \mid(f \circ g)=\left({ }_{a} \mid f\right) \circ\left({ }_{\mathbf{r}\left({ }_{a} \mid f\right)} \mid g\right)$, is that

$$
{ }_{a} \mid \mathbf{i}_{b}=\mathbf{i}_{a}=\left({ }_{a} \mid f\right) \circ\left({ }_{a f} \mid f^{-1}\right),
$$


so

$$
\left({ }_{a} \mid f\right)^{-1}={ }_{a^{f}} \mid f^{-1}, \quad a^{\mathbf{i}_{b}}=a, \quad \text { and } \quad a^{f}=\left({ }_{a f} \mid f^{-1}\right) \circ\left({ }_{a} \mid f\right) ;
$$

moreover, $a^{f \circ g}=\left(a^{f}\right)^{g}$.

We want to link right and left "actions" by $\left.(a \mid f)\right|_{b}={ }_{a} \mid\left(\left.f\right|_{b}\right)$, but there is a little problem here, since one of the two sides of that equation may fail to be defined while the other is defined. We therefore seek to extend the conditionally-defined restrictions to total maps by the following device, based on the pseudoproduct construction familiar from the ESN theorem:

For $g \in \mathscr{G}$ and any $a \in B$, define ${ }_{a}\left|g:={ }_{a \wedge \mathbf{d} g}\right| g$, the right hand side being meaningful since $a \wedge \mathbf{d} g \leq_{L} \mathbf{d} g$. (Note that if $a \leq \mathbf{d} g$ already, the notations agree.) The next figure shows the situation (where, also by extension, we write $a^{g}$ for the already-defined $\left.(a \wedge \mathbf{d} g)^{g}\right)$.

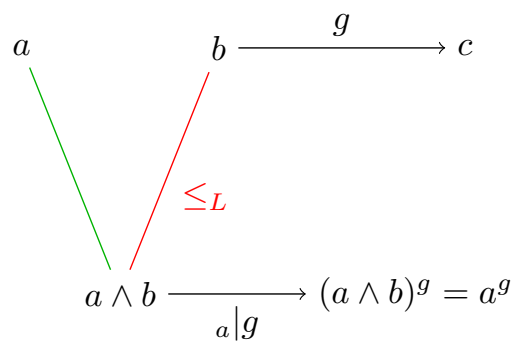

Figure 3: Generalised restriction of $g$ to object $a$ and action of $g$ on $a$.

Then if $g=\mathbf{i}_{b}$, we have $a \wedge \mathbf{i}_{b}={ }_{a \wedge b}\left|\mathbf{i}_{b}=\mathbf{i}_{a \wedge b}=\mathbf{i}_{a} \wedge \mathbf{i}_{b}=\mathbf{i}_{a} \wedge b=\mathbf{i}_{a}\right|_{a \wedge b}$, and we may write $a \wedge g$ for ${ }_{a} \mid g$ without conflict. A little re-writing of definitions shows that

$$
(a \wedge b) \wedge g={ }_{a \wedge b}\left|g={ }_{a}\right|\left({ }_{b} \mid g\right)=a \wedge(b \wedge g)
$$

and

$$
a \wedge \mathbf{d} g=\mathbf{d}(a \wedge g)=(a \wedge g) \circ(a \wedge g)^{-1} .
$$

We complete our list of postulates with the previously-mentioned $\left.\left({ }_{a} \mid f\right)\right|_{b}={ }_{a} \mid\left(\left.f\right|_{b}\right)$, which we now write as

- $(a \wedge f) \wedge b=a \wedge(f \wedge b)$, for all $a, b \in B$ and $f \in \mathscr{G}$.

(More fully, this is $\left.(a \wedge \mathbf{d} f \mid f)\right|_{a^{f} \wedge b}={ }_{a \wedge f} b \mid\left(\left.f\right|_{b \wedge \mathbf{r} f}\right)$.)

Next, we may extend the composition further, to a pseudoproduct $\otimes$ : when $f: z \rightarrow a$ and $g: b \rightarrow c$, we define

$$
f \otimes g:=\left(\left.f\right|_{a \wedge b}\right) \circ(a \wedge b \mid g)=(f \wedge(a \wedge b)) \circ((a \wedge b) \wedge g) .
$$

The pseudoproduct is defined for all pairs $f, g$.

Then $a \wedge f$ is actually just $\mathbf{i}_{a} \otimes f$. This is indeed an extension of meaning: when $f \circ g$ is defined, $f \otimes g=f \circ g$, and when $f=\mathbf{i}_{a}$ and $g=\mathbf{i}_{b}$,

$$
f \otimes g=\mathbf{i}_{a} \otimes \mathbf{i}_{b}=\mathbf{i}_{a \wedge b}=\mathbf{i}_{a} \wedge \mathbf{i}_{b}
$$

so we may as well use just the one symbol $\wedge$ for $\otimes$, as it extends $\circ$ and the restrictions, as well as the original $\wedge$ on $B$. Let us check remaining non-trivial cases for associativity. 


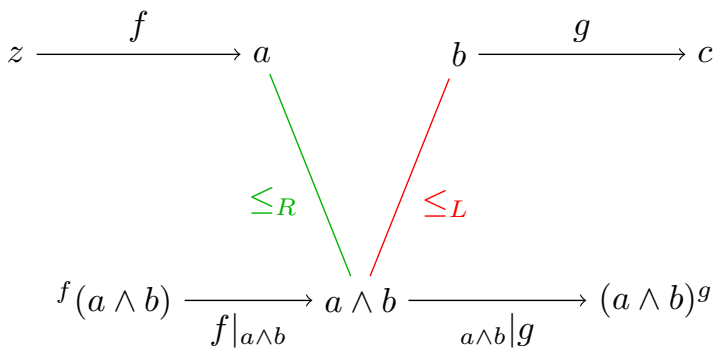

Figure 4: Diagram illustrating the pseudoproduct.

Lemma 2.1. For all $f, g \in \mathscr{G}$ and $e \in B$, with $f: \mathbf{d} f \rightarrow a$ and $g: b \rightarrow \mathbf{r} g$,

(i) $(f \wedge e) \wedge g=f \wedge(e \wedge g)$,

(ii) $e^{f \wedge g}=\left(e^{f}\right)^{g}$, and

(iii) $e \mid(f \wedge g)=(e \mid f) \wedge g$.

Proof. (i): By definition,

$$
(f \wedge e) \wedge g=\left(\left.f\right|_{e}\right) \wedge g=\left.\left(\left.f\right|_{a \wedge e}\right)\right|_{b} \circ{ }_{a \wedge e} \mid g=\left(\left.f\right|_{a \wedge e \wedge b}\right) \circ(a \wedge e \wedge b \mid g),
$$

while $f \wedge(e \wedge g)=\left.f\right|_{e \wedge b} \wedge e \wedge b \mid g=f \wedge(e \wedge g)$.

(ii): We already have $e^{f \wedge g}=e^{\left(\left.f\right|_{b}\right) \circ\left({ }_{a} \mid g\right)}=\left(e^{\left.f\right|_{b}}\right)^{(a \mid g)}$.

Observe that $e \wedge \mathbf{d}\left(\left.f\right|_{b}\right) \leq_{R} e \wedge \mathbf{d} f$, since $\leq_{R}$ is left compatible (Figure 5 may assist the reader). So $e \wedge f=\left.e \wedge f\right|_{b}$ and $e^{\left.f\right|_{b}}=e^{f}$. Likewise $e^{f} \wedge b \leq_{L} a \wedge b$ and $\left(e^{f}\right)^{g}=$ $\left(e^{f}\right)^{(a \mid g)}=e^{f \wedge g}$.

(iii): Using $e^{\left.f\right|_{b}}=e^{f}$ from (ii), we have

$$
\begin{aligned}
e \mid(f \wedge g) & ={ }_{e} \mid\left(\left.f\right|_{b} \circ{ }_{a} \mid g\right)=\left(e \mid\left(\left.f\right|_{b}\right)\right) \circ\left(e^{\left.f\right|_{b}} \mid\left({ }_{a} \mid g\right)\right) \\
& =\left(\left.(e \mid f)\right|_{b}\right) \circ\left({ }_{e^{\left.f\right|_{b} \wedge a}} \mid g\right)=\left(\left.(e \mid f)\right|_{b}\right) \circ\left(e^{f} \mid g\right) \\
& =(e \mid f) \wedge g .
\end{aligned}
$$

It remains to prove associativity in full generality:

Lemma 2.2. For all $f, g, h \in \mathscr{G}, f \wedge(g \wedge h)=(f \wedge g) \wedge h$.

Proof. First we establish that when $f \circ g$ is defined, $(f \circ g) \wedge h=f \wedge(g \wedge h)$. Let $r=\mathbf{r} g$ and $d=\mathbf{d} h$; we have

$$
\begin{aligned}
(f \circ g) \wedge h & =\left.(f \circ g)\right|_{d} \circ{ }_{r}\left|h=\left(\left.\left.f\right|_{g_{d}} \circ g\right|_{d}\right) \circ_{r}\right| h \\
& =\left.f\right|_{g_{d}} \circ\left(\left.g\right|_{d} \circ_{r} \mid h\right)=\left.f\right|_{g_{d}} \circ(g \wedge h) ;
\end{aligned}
$$

and since ${ }^{g} d=\mathbf{d}\left(\left.g\right|_{d}\right)=\mathbf{d}(g \wedge h)$, the latter is indeed $f \wedge(g \wedge h)$. Now observe that, in the general case,

$$
(f \wedge g) \wedge h=\left(\left.f\right|_{\mathbf{d} g}{ } \mathbf{r} f \mid g\right) \wedge h=\left.f\right|_{\mathbf{d} g} \wedge(\mathbf{r} f \mid g \wedge h)
$$




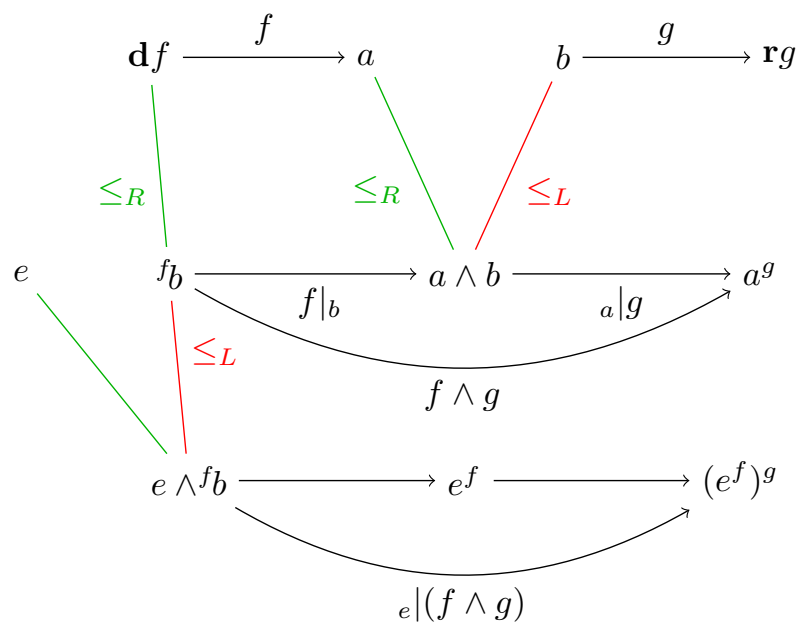

Figure 5: Diagram illustrating $e^{f \wedge g}=\left(e^{f}\right)^{g}$.

by the foregoing; and then, by Lemma 2.1(iii), we have

$$
\left.f\right|_{\mathbf{d} g} \wedge(\mathbf{r} f \mid g \wedge h)=\left.f\right|_{\mathbf{d} g} \wedge \mathbf{r} f \mid(g \wedge h)=f \wedge(g \wedge h),
$$

completing the proof.

Theorem 2.3. $S=(\mathscr{G}, \wedge)$ is an orthodox semigroup.

Proof. Lemma 2.2 shows that $S$ is a semigroup. $S$ is regular, since $g \wedge g^{-1} \wedge g=g$ for any $g \in \mathscr{G}$. If $f \wedge f=f$, then $f=\left(\left.f\right|_{b \wedge a}\right) \circ(b \wedge a \mid f)$ - see Figure 6-and in particular,

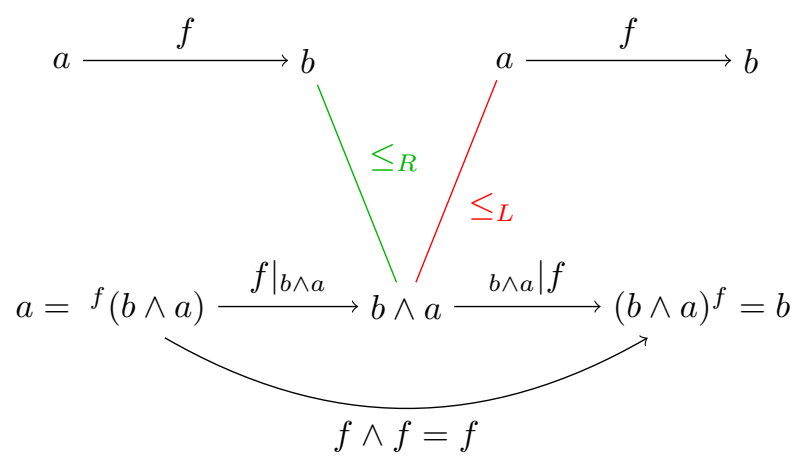

Figure 6: Diagram for an idempotent.

$a={ }^{f}(b \wedge a)$ and $b=(b \wedge a)^{f}$. Thus $(b \wedge a \mid f) \circ f^{-1}$ is defined and equal (by the composition axiom) to $\left({ }_{b \wedge a} \mid f\right) \circ\left({ }_{b} \mid f^{-1}\right)={ }_{b \wedge a}\left|\left(f \circ f^{-1}\right)={ }_{b \wedge a}\right| \mathbf{i}_{a}=\mathbf{i}_{b \wedge a}$. So we have $\mathbf{i}_{a}=f \circ f^{-1}=$ $\left(\left.f\right|_{b \wedge a}\right) \circ \mathbf{i}_{b \wedge a}=\left.f\right|_{b \wedge a}$, giving $f=\mathbf{i}_{a}$. Thus $E(S)=B$ and $S$ is orthodox.

It also follows that every idempotent is of the form $f \wedge f^{-1}$. With $s \in S$, put $s^{+}=$ $s \wedge s^{-1}$ and $s^{-}=s^{-1} \wedge s$. Clearly $\left(s^{-1}\right)^{+}=s^{-}$and $\left(s^{-1}\right)^{-}=s^{+}$, while $s^{+} \mathscr{R} s \mathscr{L} s^{-}$ 
( $\mathscr{R}$ and $\mathscr{L}$ being the usual Green's relations in $S$ ) and $s^{-1}$ is the unique inverse of $s$ such that $s^{+} \mathscr{L} s^{-1} \mathscr{R} s^{-}$.

Theorem 2.4. For all $s, t \in S$, there hold:

(i) $s^{+} \wedge s^{+}=s^{+}=\left(s^{+}\right)^{+}=\left(s^{+}\right)^{-}$and $s^{-} \wedge s^{-}=s^{-}=\left(s^{-}\right)^{-}=\left(s^{-}\right)^{+}$;

(ii) $s^{+} \wedge s=s=s \wedge s^{-}$;

(iii) $s \wedge s=s$ implies $s=s^{+}=s^{-}$;

(iv) $(s \wedge t)^{+}=\left(s \wedge t^{+}\right)^{+}$and $(s \wedge t)^{-}=\left(s^{-} \wedge t\right)^{-}$;

(v) $\left(s^{+} \wedge t\right)^{+}=s^{+} \wedge t^{+}$and $\left(s \wedge t^{-}\right)^{-}=s^{-} \wedge t^{-}$.

Proof. Parts (i) - (iii) follow by easy computation from the definitions and Theorem 2.3. The definition of the extended $\wedge$ in the new notation (see Figure 7) reads $s \wedge t=\left(s \wedge t^{+}\right) \circ$ $\left(s^{-} \wedge t\right)$, and (iv) follows immediately. Part (v) is a consequence of (iv) with, respectively, $s^{+}$for $s$ and $t^{-}$for $t$.

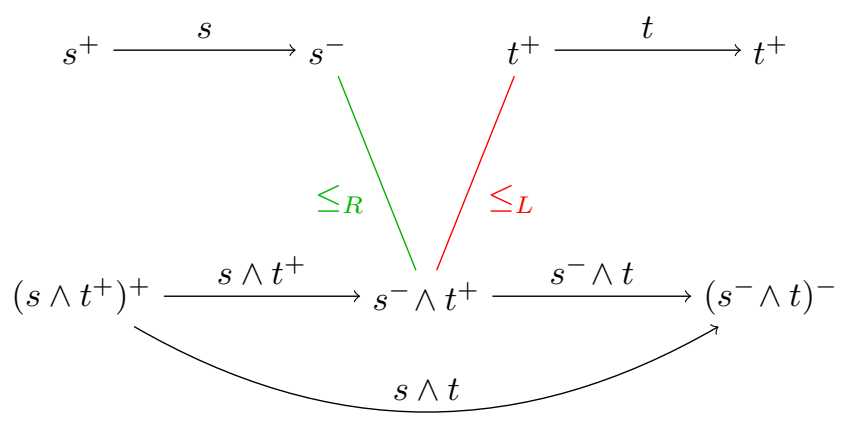

Figure 7: The pseudoproduct in $+/-$ notation.

Remarks 2.5. Theorem 2.4 sets out the object part of a functor imitating that of the ESN theorem. There may be another occasion to describe the morphism part, which should also involve examining the properties in the Theorem, since they include some of those forming the definitions of restriction and Ehresmann semigroups. In fact, $(s \wedge t)^{+} \wedge s=s \wedge t^{+}$ and $t \wedge(s \wedge t)^{-}=s^{-} \wedge t$ hold in a restriction semigroup as defined by Kudryavtseva [6], but fail here unless $B$ is a semilattice (in which case $S$ is inverse). The restriction and Ehresmann classes are surveyed in [1], and one may see the directions in which the ideas have been taken more recently in [5] and [6]. This strand of research emphasises commuting idempotents, which distinguishes them from the present paper, where an element may have multiple left and right identities. This may appear a little strange, but is the price to be paid for dealing with all idempotents, not just a special subset. More general contexts have already been considered, as in $[2,12,13]$, but the approach in hand is a natural and minimal extension of the inductive groupoid case, and returns to the spirit of groupoids as dealt with in another landmark paper-Lawson's [7]. 
Above all, our ultimate intent is to have $B$ as a skew lattice. We deal with this in the next section, using the results above: beginning with a skew lattice $B=(B, \wedge, \vee)$, we dualise the whole process of Section 2 to extend the join operation $\vee$ to $\mathscr{G}$, resulting in an algebra $S=(\mathscr{G}, \vee, \wedge)$.

\section{Skew lattices of objects}

Let $\mathscr{G}$ be a groupoid with composition $\circ$ and $B$ its set of objects, endowed with associative operations $\vee$ and $\wedge$ satisfying the absorptive axioms

$$
a \vee(a \wedge b)=a=a \wedge(a \vee b), \quad(a \wedge b) \vee b=b=(a \vee b) \wedge b
$$

for a skew lattice $[10,11]$. Then both $(B, \vee)$ and $(B, \wedge)$ are bands. Moreover each has a pair of natural preorders: in the lower band $(B, \wedge)$ we write (continuing on from the preceding Section 2)

- $a \leq_{L} b$ if and only if $a=a \wedge b$, and $a \leq_{R} b$ if and only if $a=b \wedge a$,

and additionally in the upper band $(B, \vee)$ we write

- $a \geq_{L} b$ if and only if $a=a \vee b$, and $a \geq_{R} b$ if and only if $a=b \vee a$.

We do not at this stage admit the usual convention that $\leq$ and $\geq$ are converse relations! The skew lattice absorptive axioms imply that $a=a \vee b \Longleftrightarrow a \wedge b=b$ and $a \vee b=b \Longleftrightarrow$ $a=a \wedge b$, so that $a \leq_{L} b$ if and only if $a=a \wedge b$ if and only if $b \geq_{R} a$; which is to say that $\leq_{L}$ and $\geq_{R}$ are converse relations, as also $\leq_{R}$ and $\geq_{L}$. We write the relations in the form most suitable to the occasion. As a vertical dual to the set-up in Section 2, we postulate left and right extension operations denoted ${ }^{a}|,|^{a}: \mathscr{G} \rightarrow \mathscr{G}$ such that

- ${ }^{a} \mid g$ is defined whenever $a \geq_{L} \mathbf{d} g$, and ${ }^{a} \mid g: a \rightarrow \mathbf{r}\left({ }^{a} \mid g\right) \geq_{L} \mathbf{r} g$;

and again (lateral-) dually,

- $\left.g\right|^{a}$ is defined whenever $a \geq_{R} \mathbf{r} g$, with $\left.g\right|^{a}: \mathbf{d}\left(\left.g\right|^{a}\right) \rightarrow a, \mathbf{d}\left(\left.g\right|^{a}\right) \geq_{L} \mathbf{d} g$.
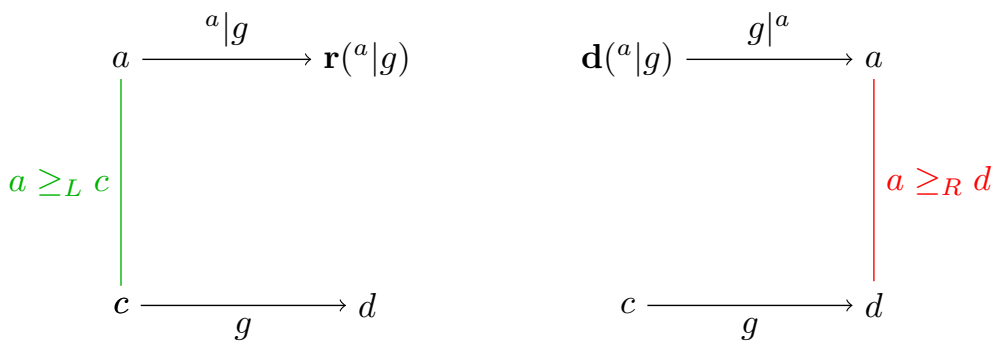

Figure 8: Left and right extension operators.

The relevant diagrams appear in Figure 8. Again we are able to write $a \vee g$ for ${ }^{a} \mid g$ and by extension for ${ }^{a \vee \mathbf{d} g} \mid g$, and $a_{g}$ for $\mathbf{r}\left({ }^{a} \mid g\right)$; similarly, $g a=\mathbf{d}\left({ }^{a} \mid g\right)$.

The postulates vertically dual to those of the preceding Section 2 are to hold also, and we list them here, using the abbreviated notation developed in Section 2 and without further 
explanation; moreover we only give one-sided forms, assuming the lateral duals hold by implication. Thus each postulate stands for a quartet (although some are self-dual or may have vertical and lateral duals equivalent).

(i) (identities) $\mathbf{d} g \vee g=g$;

(ii) (preorders) if $a \geq_{R} b$, then $a \vee \mathbf{i}_{b}=\mathbf{i}_{a \vee b}$;

(iii) (transitivity) if $a \geq_{R} b \geq_{R} \mathbf{d} g$, then $a \vee g=(a \vee b) \vee g=a \vee(b \vee g)$;

(iv) (composition) if $f \circ g$ is defined (so that $\mathbf{r} f=\mathbf{d} g$ ), then

$$
a \vee(f \circ g)=(a \vee f) \circ\left(a_{f} \vee g\right) \text {; }
$$

(v) (dual of Theorem 2.3) $\quad(a \vee f) \vee b=a \vee(f \vee b)$, for all $a, b \in B$ and $f \in \mathscr{G}$.

The vertical dual of the development in Section 2 extends the join operation $\vee$ to all of $\mathscr{G}$ and of course the dual results hold. In particular, we note that

$$
a \vee f \vee f^{-1}=a \vee f \vee(a \vee f)^{-1} \text {. }
$$

Moreover, extra postulates are required to establish compatibility conditions between the restriction and extension operators which reflect the skew lattice character of $B$.

Observe that when $f \circ g$ is defined,

$$
f \vee g=f \wedge g=f \circ g
$$

in particular, $f \vee f^{-1}=f \wedge f^{-1}=\mathbf{d} f$, etc. From this point on, we write (to conform to precedent) $f^{*}$ in place of $f^{-1}$, and may as well write $f f^{*}$ for $f \circ f^{*}=f \wedge f^{*}=f \vee f^{*}$, etc. The identification of $\mathbf{i}_{a}$ with $a$ also identifies $a^{*}$ with $\mathbf{i}_{a}^{-1}=\mathbf{i}_{a}$ and so $(a \wedge f)^{*}$ with $a^{f} \wedge f^{*}$, and similarly $(a \vee f)^{*}=a^{f} \vee f^{*}$.

The restriction and extension operators should also be linked through the skew lattice orders. Consider any object $a \in B$ and morphism $f$; write $\mathbf{d} f=d=f f^{*}$ and $\mathbf{r} f=$ $r=f^{*} f$, and set $b=r \vee a \geq_{R} r$. Then ${ }_{a} \mid f: a \rightarrow a^{f}$ exists, and $a^{f} \leq_{L} r$, which is to say $r \geq_{R} a^{f}$, and so there is $\left.\left({ }_{a} \mid f\right)\right|^{r}: d^{\prime} \rightarrow r$. When $f=\mathbf{i}_{d}$, we see that $d^{\prime}=d$ so it is reasonable that this hold in general. See Figure 9.

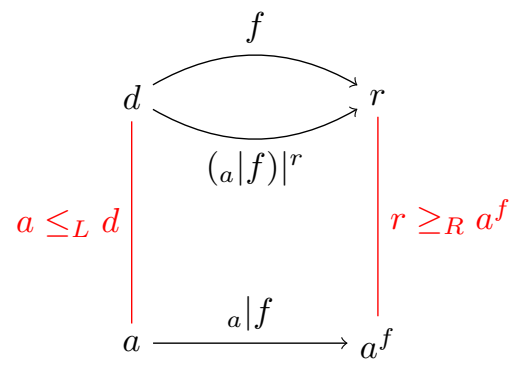

Figure 9: Restriction and extension operators linked.

Indeed we shall require, as a linking condition, that $\left.\left({ }_{a} \mid f\right)\right|^{r}=f$ and so we add to the previous list the axiom 
(vi) $f=(a \wedge f) \vee f^{*} f$, or equivalently $f f^{*}=(a \wedge f) \vee f^{*}$.

We also assume the lateral and order duals, which are interpreted similarly. Note that when $f \in B, f=f^{*}=f f^{*}$, and this equation reduces to the absorptive identity

$$
f=(a \wedge f) \vee f
$$

of skew lattices.

We shall (tentatively) refer to a groupoid satisfying these conditions as a skew inductive groupoid. Theorem 2.3 applies and assures the existence of an algebra $(S, \vee, \wedge, *)$ arising from a skew inductive groupoid. We now seek to characterise such an algebra axiomatically.

\section{Algebraic characterisation}

Let $(S, \vee, \wedge, *)$ be an algebra of signature $(2,2,1)$, with $\vee, \wedge: S \times S \rightarrow S$ and ${ }^{*}: S \rightarrow S$, that satisfy, for all $s, t \in S$ :

(i) $(S, \vee)$ and $(S, \wedge)$ are associative (thus, semigroups);

(ii) $\left(s^{*}\right)^{*}=s$;

(iii) $s \vee s^{*}=s \wedge s^{*}=\left(s \wedge s^{*}\right)^{*}$;

(iv) $s \vee s^{*} \vee s=s=s \wedge s^{*} \wedge s$;

(v) $s \vee s=s$ or $s \wedge s=s$ implies $s=s^{*}$;

(vi) $s \vee s^{*} \vee\left(s \wedge t^{*} \wedge t\right)=s=s \wedge s^{*} \wedge\left(s \vee t^{*} \vee t\right)$ and lateral duals;

(vii) $s \vee s^{*} \vee t \vee t^{*}=s \vee s^{*} \vee t \vee\left(s \vee s^{*} \vee t\right)^{*}$ and duals;

(viii) $s^{*} \vee s=t \vee t^{*}$ implies

$$
\begin{aligned}
& s \vee t \vee(s \vee t)^{*}=s \vee s^{*} \quad \text { and } \quad(s \vee t)^{*} \vee s \vee t=t^{*} \vee t \quad \text { and } \\
& s \wedge t \wedge(s \wedge t)^{*}=s \wedge s^{*} \quad \text { and } \quad(s \wedge t)^{*} \wedge s \wedge t=t^{*} \wedge t \text {. }
\end{aligned}
$$

The properties in Section 3, particularly Lemmas 2.1, 2.2 and Theorem 2.3, show that we were able to construct such an object from a skew inductive groupoid. Conversely, we have

Theorem 4.1. Let $(S, \vee, \wedge, *)$ satisfy axioms $(i)-(v i i i)$, and form a small category $\mathscr{C}$ as follows.

- $\mathrm{Ob}(\mathscr{C})=\left\{s \vee s^{*}: s \in S\right\}$,

- $\operatorname{Mor}(\mathscr{C})=\left\{\widehat{s}=\left(s \vee s^{*}, s, s^{*} \vee s\right): s \in S\right\}$,

- when $s^{*} \vee s=\mathbf{r}(\widehat{s})=\mathbf{d}(\widehat{t})=t \vee t^{*}, \widehat{s} \circ \widehat{t}$ is defined and

$$
\widehat{s} \circ \widehat{t}=\left(s \vee s^{*}, s t, t^{*} \vee t\right) .
$$


Then $\mathscr{C}$ is a skew inductive groupoid whose pseudoproduct gives an orthodox semigroup isomorphic with $S$.

Proof. From (vi) we have that $\mathrm{Ob}(\mathscr{C})$ is a skew lattice. Clearly composition when defined for triples is associative, and each $\left(s \vee s^{*}, s \vee s^{*}, s^{*} \vee s\right)$ is the identity at object $s \vee s^{*}$. Morphism $\widehat{s}=\left(s \vee s^{*}, s, s^{*} \vee s\right)$ has inverse $\widehat{s}^{-1}=\left(s^{*} \vee s, s^{*}, s \vee s^{*}\right)$. The restriction and extension operators must be defined: for a morphism $\widehat{s}=\left(s \vee s^{*}, s, s^{*} \vee s\right)$ and an object $a$ such that $a \geq_{L} s \vee s^{*}$ (i.e. $a=a \vee s \vee s^{*}$ ), we set

$$
{ }^{a} \mid \widehat{s}=\left(a, a \vee s,(a \vee s)^{*} \vee a \vee s\right)
$$

The r.h.s. is indeed in $\operatorname{Mor}(\mathscr{C})$ : by equation (2.2), $(a \vee s) \vee(a \vee s)^{*}=(a \vee s) \vee s^{*}=a$ by hypothesis. Moreover,

$$
\mathbf{r}\left({ }^{a} \mid \widehat{s}\right) \vee s \vee s^{*}=\left((a \vee s)^{*} \vee a \vee s\right) \vee s \vee s^{*}=\mathbf{r}\left({ }^{a} \mid \widehat{s}\right),
$$

so $\mathbf{r}\left({ }^{a} \mid \widehat{s}\right) \geq_{L} \mathbf{r}(s)$, as required for an extension operator.

Next, the postulates of Section 3 have to be verified. It is useful to observe that the right [left] component of a left- [right-]extended morphism depends solely on the middle component, and so may safely be left unspecified (written $\sim$ ) in certain calculations.

(i) ("identity") follows from regularity (axiom (iv)).

(ii) ("preorder") Assume $a=b \vee a$. By definition,

$$
{ }^{a}\left|\mathbf{i}_{b}={ }^{(a, a, a)}\right|(b, b, b)=\left(a \vee b, a \vee b,(a \vee b)^{*} \vee a \vee b\right)=\mathbf{i}_{a \vee b}
$$

(iii) ("transitivity") First, ${ }^{b}|\widehat{s}=(b, b, b)|\left(s \vee s^{*}, s, s^{*} \vee s\right)=\left(b \vee s \vee s^{*}, b \vee s, \sim\right)$, so

$$
{ }^{a}\left|\left({ }^{b} \mid \widehat{s}\right)={ }^{a}\right|\left(b \vee s \vee s^{*}, b \vee s, \sim\right)=\left(a \vee b \vee s \vee s^{*}, a \vee b \vee s, \sim\right)={ }^{(a \vee b)} \mid \widehat{s},
$$

by associativity of $S$.

(iv) ("composition")

$$
\begin{aligned}
\left.\left({ }^{a} \mid \widehat{s}\right)\right|^{b} & =\left.\left(a \vee s \vee s^{*}, a \vee s,(a \vee s)^{*} \vee a \vee s\right)\right|^{b} \\
& =\left((a \vee s \vee b)(a \vee s \vee b)^{*}, a \vee s \vee b,(a \vee s)^{*} \vee a \vee s \vee b\right),
\end{aligned}
$$

while

$$
\begin{aligned}
{ }^{a} \mid\left(\left.\widehat{s}\right|^{b}\right) & ={ }^{a} \mid\left((s \vee b)(s \vee b)^{*}, s \vee b,(s \vee s)^{*} \vee b\right) \\
& =\left(a \vee(s \vee b)(s \vee b)^{*}, a \vee s \vee b,(a \vee s \vee b)^{*} \vee a \vee s \vee b\right),
\end{aligned}
$$

and by (the lateral dual of) axiom (vii), these are equal.

(v) (“dual of Theorem 2.3") This follows from associativity in $S$.

In this manner we have constructed a groupoid $\widehat{S}$ over a skew lattice of objects. Now suppose that $S$ arises from the original groupoid $\mathscr{G}$. The mapping $\mathscr{G} \rightarrow \widehat{S}$ given by $g \mapsto$ $(\mathbf{d} g, g, \mathbf{r} g)$ is routinely an isomorphism, simply representing different ways of describing $\mathscr{G}$; the fact that it factors through $S$ completes the proof. 


\section{Models}

Do such objects even exist? One special case occurs with $\mathscr{G}$ a true inductive groupoid and $B$ a lattice. Such a combination gives rise to two inverse semigroups (monoids in fact), and an easy way to realise such an object is by taking the direct product of a group with a lattice. This could be the inspiration for a less trivial example, as follows.

Let a group $G$ act by automorphisms on a band $B$. Then we may consider the semidirect product $S=G \ltimes B$ with base set $G \times B$ and multiplication, for $u, v \in G$ and $a, b \in B$,

$$
(u, a)(v, b)=\left(u v, a^{v} \cdot b\right)
$$

This situation was studied some time ago by Miklós Hartmann and Mária Szendrei [3, 4] and maybe others I have not yet found; and it seems to have been generalised in [2]. All we need to note here is that

- idempotents are exactly the elements $(1, a)$, and $E(S) \cong B$,

- $S$ is regular with an involution $(u, a)^{*}=\left(u^{-1}, a^{u^{-1}}\right)$, such that

- $(u, a)(u, a)^{*}=(u, a)\left(u^{-1}, a^{u^{-1}}\right)=\left(1, a^{u^{-1}}\right), \quad(u, a)^{*}(u, a)=(1, a)$

- $(u, a)(u, a)^{*}(u, a)=(u, a)$

- so $S$ is orthodox but not inverse

- and $*$ is not an anti-automorphism.

On this last point, let us observe that $[(u, a)(v, b)]^{*}=\left(v^{-1} u^{-1}, a^{u^{-1}} \wedge b^{v^{-1} u^{-1}}\right)$, so

$$
[(u, a)(v, b)]^{*}[(u, a)(v, b)]=\left(1,\left(a \wedge b^{v^{-1}}\right)^{u^{-1}}\right),
$$

which reduces to $\left(1, a^{u^{-1}}\right)$ precisely when $a=b^{v^{-1}}$, i.e., when

$$
(u, a)^{*}(u, a)=(v, b)(v, b)^{*} .
$$

In structural terms, these are both equivalent to $(u, a) \mathscr{R}(u, a)(v, b) \mathscr{L}(v, b)$. (This may also be relevant to criteria for composibility in the double-orthodox semigroup set-up.)

We may conventionally write a "normal form" $u a$ for $(u, a)$. Then $(u, a)=(u, \top)(1, a)$ when $B$ has a top element $\top$, and so $S=G B$ and we have the factorisable case. Otherwise, $S \cup G$ is factorisable and $S$ almost factorisable. See also Rida-e Zenab's recent article [14], and its references, for Zappa-Szép products of which this is also an example.

The map $\phi: S \rightarrow G, u a \mapsto u$ partitions $S$ into blocks $S_{u}=u \phi^{-1}$, and $S_{u}$ is isomorphic with $B$ when given the sandwich multiplication (for $u a, u b \in S_{u}$ ), $u a \star u b=$ $u a\left(u^{-1}\right) u b=u a b$; so $S$ is a "group of (isomorphic) sandwich bands". Conversely, given such a $\left\{B_{u}: u \in G\right\}$ with connecting isomorphisms

$$
\left\{\lambda_{u, v}, \rho_{u, v}: B_{u} \rightarrow B_{v}\right\}
$$

satisfying the right axioms, one may reconstruct $S=\bigcup B_{u}$ with multiplication (for $s \in$ $B_{u}, t \in B_{v}$ ) given by

$$
s \cdot t=s \rho_{u, u v} \star t \lambda_{v, u v}
$$


with $\star$ the multiplication in $B_{u v}$. (There is nothing special about this, it's just another description of a semidirect product.)

Then we can see what happens when we do it twice over, replacing · by $\wedge$ and $\vee$. (We will end up with an algebra of signature $(2,2,1)$.) Note that $(u, a)=u \wedge a=u \vee a$ in the normal form, and

$$
\begin{aligned}
& u a \wedge(u a)^{*}=u a \wedge u^{-1} a^{u^{-1}}=1 \wedge a^{u^{-1}} \\
& (u a)^{*} \wedge u a=u^{-1} a^{u^{-1}} \wedge u a=1 \wedge a
\end{aligned}
$$

and exactly the same with the $\vee$ operation. Thus $s \vee s^{*}=s \wedge s^{*}=s s^{*}$, etc. (using juxtaposition where either main operation may be applied). So the absorptive identity $a \vee(a \wedge b)=a$ is equivalent to

$$
s^{*} s \vee\left(s^{*} s \wedge t^{*} t\right)=s^{*} s \quad \text { and so to } \quad s \vee\left(s^{*} s \wedge t^{*} t\right)=s ;
$$

and likewise for the lateral and order duals.

The theory of inverse semigroups suggests that we investigate an idempotent-separating *-congruence $\sim$ of such a $G \ltimes B$. If $u a \sim v b$ then $a \sim b=a$; so we are led to consider the subgroups $K_{a}:=\{u \in G: u a=a\}$. Now

$$
K_{a} \subseteq K_{a \vee b} \subseteq K_{(a \vee b) \wedge b}=K_{b}
$$

for all $a, b \in B$; thus $K_{a}=K \unlhd G$, say; and we may as well have started with $G / K$.

The groupoid version of $G \ltimes B$ may be presented as follows. Given a skew lattice $B$ and a group $G$ acting by automorphisms on $B$, make a category with objects from $B$ and morphisms $\left(b, g, b^{g}\right)$. The composition $\left(b, g, b^{g}\right) \circ\left(c, h, c^{h}\right)$ is defined exactly when $b^{g}=c$, and is given by $\left(b, g, b^{g}\right) \circ\left(c, h, c^{h}\right)=\left(b, g h, b^{g h}\right)$. If one works it through, one has the pseudoproduct

$$
\left(b, g, b^{g}\right) \otimes_{\wedge}\left(c, h, c^{h}\right)=\left(b \wedge c^{g-1}, g h, b^{g h} \wedge c^{h}\right),
$$

which we may abbreviate $\left(g, b^{g}\right) \cdot\left(h, c^{h}\right)=\left(g h, b^{g h} \wedge c^{h}\right)$, the semidirect product.

\section{Further comments}

The restriction idea may provide another useful way of thinking about skew lattices. It remains to describe categories of orthodox semigroups with involutory inversion $*$ and of skew inductive groupoids, and functors establishing an equivalence between them. Refinement of the axioms may also be possible, and the relationships with the approach of actions (of objects on morphisms and morphisms on objects) should be explored. The connexions with restriction and Ehresmann semigroups need to be teased out. More "natural" or concrete examples would be desirable_-for example, can they be found in rings or override algebras?

\section{References}

[1] V. Gould, Notes on restriction semigroups and related structures, 2010, manuscript, http: //www-users.york.ac.uk/ varg1/restriction.pdf.

[2] V. Gould and Y. Wang, Beyond orthodox semigroups, J. Algebra 368 (2012), 209-230, doi: 10.1016/j.jalgebra.2012.06.012. 
[3] M. Hartmann, Almost factorizable orthodox semigroups, Semigroup Forum 74 (2007), 106124, doi:10.1007/s00233-006-0618-1.

[4] M. Hartmann and M. B. Szendrei, E-unitary almost factorizable orthodox semigroups, Semigroup Forum 84 (2012), 157-175, doi:10.1007/s00233-011-9299-5.

[5] P. R. Jones, A common framework for restriction semigroups and regular *-semigroups, J. Pure Appl. Algebra 216 (2012), 618-632, doi:10.1016/j.jpaa.2011.07.014.

[6] G. Kudryavtseva, Two-sided expansions of monoids, Internat. J. Algebra Comput. 29 (2019), 1467-1498, doi:10.1142/s0218196719500590.

[7] M. V. Lawson, Semigroups and ordered categories. I. The reduced case, J. Algebra 141 (1991), 422-462, doi:10.1016/0021-8693(91)90242-z.

[8] M. V. Lawson, Inverse Semigroups: The Theory of Partial Symmetries, World Scientific, River Edge, New Jersey, 1998, doi:10.1142/9789812816689.

[9] M. V. Lawson and P. Scott, AF inverse monoids and the structure of countable MV-algebras, $J$. Pure Appl. Algebra 221 (2017), 45-74, doi:10.1016/j.jpaa.2016.05.025.

[10] J. Leech, Skew lattices in rings, Algebra Universalis 26 (1989), 48-72, doi:10.1007/ bf01243872.

[11] J. Leech, Recent developments in the theory of skew lattices, Semigroup Forum 52 (1996), 7-24, doi:10.1007/bf02574077.

[12] M. B. Szendrei, Embedding into almost left factorizable restriction semigroups, Comm. Algebra 41 (2013), 1458-1483, doi:10.1080/00927872.2011.643839.

[13] M. B. Szendrei, Embedding of a restriction semigroup into a $W$-product, Semigroup Forum 89 (2014), 280-291, doi:10.1007/s00233-013-9516-5.

[14] R. Zenab, Algebraic properties of Zappa-Szép products of semigroups and monoids, Semigroup Forum 96 (2018), 316-332, doi:10.1007/s00233-017-9878-1. 